\title{
Étude ethnobotanique des plantes médicinales utilisées dans le traitement du diabète dans le Département de Zouénoula (Côte d'Ivoire)
}

\author{
Arthur Stéphane GNAGNE¹, Djeneb CAMARA1, N'Guessan Bra Yvette FOFIE², Kouadio BENE ${ }^{1^{*}}$ et Guédé \\ Noël ZIRIHI ${ }^{1}$ \\ ${ }^{1}$ Laboratoire de Botanique, UFR Biosciences, Université Félix Houphouët Boigny de Cocody Abidjan ; 22 BP 582 \\ Abidjan 22 \\ 2Laboratoire de Pharmacognosie Botanique et Cryptogamie, UFR Sciences Pharmaceutiques et Biologiques, \\ Université Félix Houphouët-Boigny, Abidjan ; 22 BP 582 Abidjan 22. *Auteur correspondant : \\ kouadio777@gmail.com
}

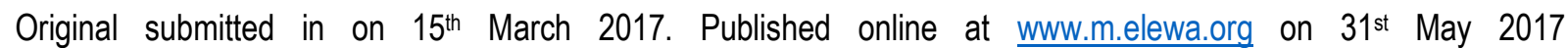
https://dx.doi.org/10.4314/jab.v113i1.14

\section{RÉSUMÉ}

Objectif : Le but de cette étude est de contribuer à une meilleure connaissance des plantes médicinales antidiabétiques utilisées dans le Département de Zouénoula.

Méthodologie et résultats : Des enquêtes ethnobotaniques ont été réalisées à l'aide de questionnaires auprès des guérisseurs du Département. Les résultats obtenus ont permis d'inventorier dix-sept espèces de plantes médicinales appartenant à quatorze familles botaniques et réparties en seize genres. Parmi ces espèces, Ageratum conyzoides (Herbe aux sorciers), Anthocleista djalonensis (Arbre chou) et Bidens pilosa (Piquant noir) sont les plus recommandées. Les feuilles sont les organes les plus utilisés. La décoction est le mode de préparation le plus sollicité et l'administration se fait majoritairement par voie orale.

Conclusion et application des résultats: Ce travail constitue une source d'informations pouvant servir de base pour des études pharmacologiques afin d'évaluer l'efficacité thérapeutique et l'innocuité de ces trois plantes à effet antidiabétique traditionnel.

Mots- clés : Ethnobotanique, plantes médicinales, diabète, Zouénoula.

\begin{abstract}
Objective: The aim of this study is to contribute to a better knowledge of medicinal antidiabetic herbs used in the Zouénoula Department.

Methodology and results: Ethnobotanical surveys were carried out using questionnaires from the Department's healers. The results obtained made it possible to inventory seventeen species of medicinal plants belonging to fourteen botanical families and divided into sixteen genera. Among these species, Ageratum conyzoides (Goatweed), Anthocleista djalonensis (Cabbage tree) and Bidens pilosa (Black jack) are the most recommended. The leaves are the most used organs. The decoction is the mode of preparation most requested and the administration is done mainly by oral way.

Conclusion and application of results: This work constitutes a source of information which can serve as a basis for pharmacological studies in order to evaluate the therapeutic efficacy and safety of these three plants with traditional antidiabetic effect.
\end{abstract}


Keywords: Ethnobotanical survey, medicinal plants, diabetes, Zouenoula.

\section{INTRODUCTION}

Le diabète est une affection chronique plurifactorielle se traduisant par un taux de sucre élevé dans le sang. La valeur normale de la glycémie à jeun est comprise entre 0,8 à 1,2 grammes par litre. En dessous de ces valeurs, on est en hypoglycémie, et au-dessus on est en hyperglycémie. Cette anomalie métabolique est due à une insuffisance ou à une mauvaise utilisation de l'insuline par l'organisme (Ghourri et al., 2013). En 2013, on estimait que près de 20 millions de personnes étaient atteintes de diabète en Afrique Subsaharienne, soit une prévalence de $4,9 \%$. D'ici à 2035 , ces estimations devraient doubler et atteindre 41,5 millions, soit une augmentation de $109 \%$ (SANOFI, 2014). Pour la Côte d'Ivoire, la Fédération Internationale du Diabète (FID) a estimé en 2014, la prévalence du diabète sucré à $9,6 \%$, ce qui est un problème majeur de santé publique (Kroa et al., 2016). Le diabète et ses complications ont des répercussions socioéconomiques importantes. Cette maladie peut endommager les vaisseaux sanguins, les nerfs, les yeux et les reins. Le diabète peut entraîner une hypertension artérielle et perturber l'activité

\section{MATÉRIEL ET MÉTHODES}

Matériel végétal: Ce sont l'ensemble des plantes médicinales recensées dans le Département de Zouénoula.

\section{Méthodes d'étude}

Enquête ethnobotanique : Avant de sortir sur le terrain pour mener l'étude ethnobotanique proprement dite, nous avons procédé à la localisation des différents sites d'enquêtes dans le Département étudié. Ensuite des enquêtes, basées sur les interrogations directes portant sur les usages des plantes citées dans la pharmacopée traditionnelle, ont été conduites auprès de la population locale. Pour avoir un inventaire ethnobotanique varié d'une zone à une autre dans la zone étudiée et le plus complet possible, l'enquête a été réalisée à l'aide d'un questionnaire.

Échantillonnage : Selon le découpage administratif de 2012, le Département de Zouénoula est composé de sept Sous-préfectures. Dans cette étude, l'échantillon est élaboré grâce à un mode d'échantillonnage probabiliste (aléatoire), il est divisé en groupes ou strates, on a donc quatre strates $\left(S_{n}\right)$ : $\mathbf{S 1}$ (Sous-préfecture de Iriéfla cardiaque. Les coûts élevés des traitements classiques orientent les diabétiques vers les remèdes traditionnels (OMS, 2011). Par ailleurs, la prise en charge médicale du diabète est limitée par l'inaccessibilité de certaines populations aux centres de santés. Dans ces conditions les populations font souvent recours aux plantes médicinales pour se soigner (Fah et al., 2013). Ces thérapeutiques traditionnelles ayant des compositions chimiques et des propriétés pharmacologiques méconnues sont de plus en plus proposées aux diabétiques. II apparait donc primordial pour les scientifiques de réaliser des études ethnobotaniques, phytochimiques et pharmacologiques afin de valider les vertus thérapeutiques accordées à ces préparations. À la lumière de l'ensemble des résultats obtenus, des Médicaments Traditionnels Améliorés accessibles à tous seront mis à la disposition des populations. La présente étude a été initiée en vue de cataloguer les plantes médicinales sollicitées dans le traitement du diabète dans le Département de Zouénoula en Côte d'Ivoire.

Vouéboufla), S2 (Gohitafla et Maminigui), S3 (Souspréfecture de Zouénoula) et $\mathbf{S 4}$ (Zanzra et Kanzra) (Figure 1). Afin d'avoir une meilleure représentativité du Département, nous avons procédé à un échantillonnage aléatoire stratifié proportionnel. Des échantillons de 25 personnes sont ensuite formés pour chacune des quatre strates et sont mis ensemble pour constituer l'échantillon global. Ce dernier est composé de 100 acteurs de la médecine traditionnelle qui répondent aux principales caractéristiques de la population du Département de Zouénoula (répartition selon le genre, l'âge et le niveau d'instruction) (Tahri et al., 2012). Dans le but d'assurer une grande objectivité des données obtenues de notre étude, l'enquête est réalisée à l'aide d'une fiche d'enquête ou questionnaire (Salhi et al., 2010). Dans la présente étude, la nomenclature des espèces végétales suit celle de la troisième version de classification botanique des angiospermes établie par l'Angiosperm Phylogeny Group (APG III), Chase et Reveal (2009) et APG III, (2009). 


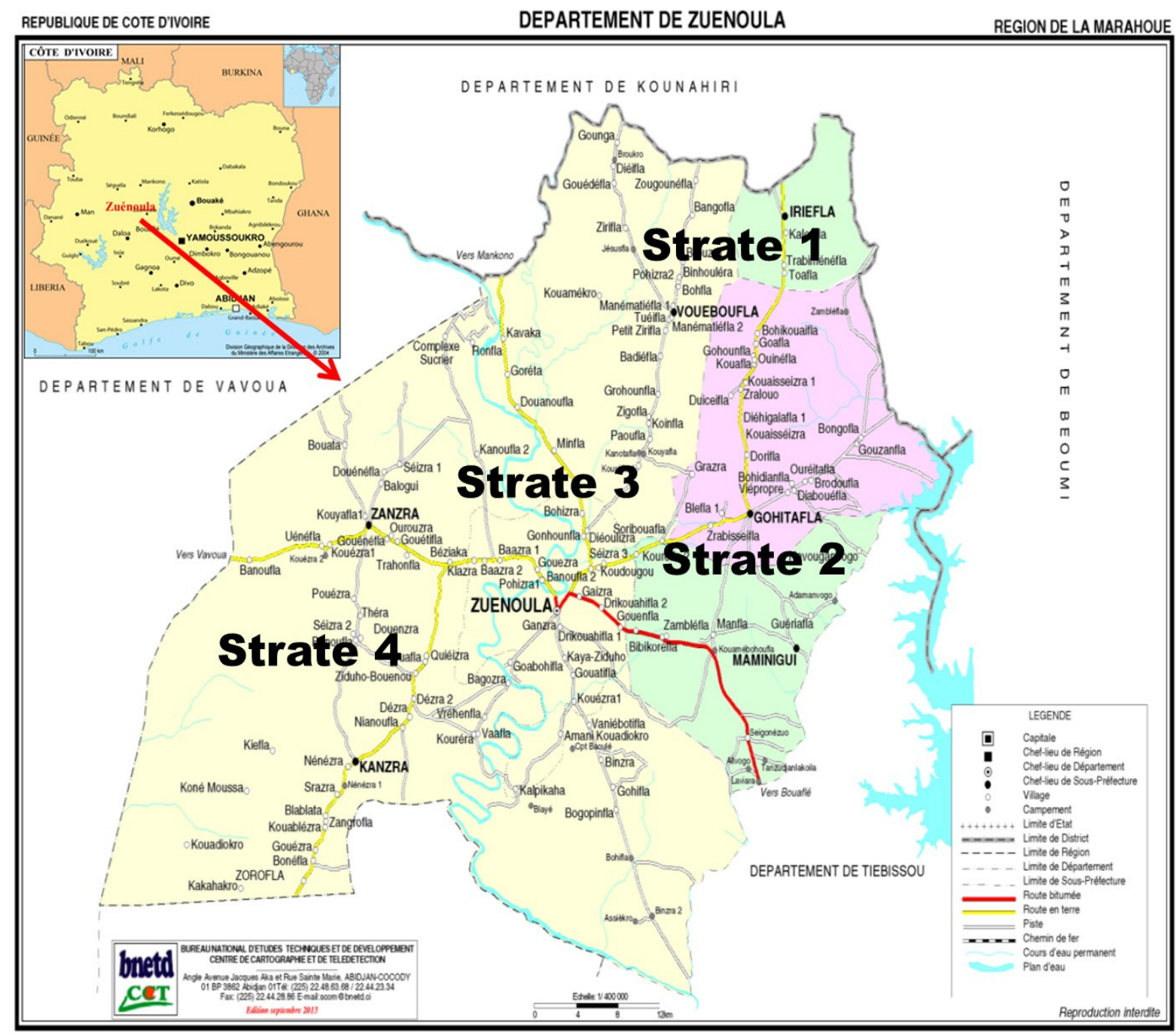

Figure 1 : Carte de localisation des sites d'enquêtes ethnobotaniques

Sélection des espèces : Deux critères de sélection ont guidé le choix des espèces médicinales antidiabétiques. Le premier critère s'est basé sur les trois premières plantes les plus sollicitées dans le traitement du diabète (fréquence de citation) et le second critère observé a été la disponibilité (l'abondance) de ces taxons sur les sites rencontrés. La fréquence de citation $(F C)$ traduit la régularité dans la distribution d'une espèce au sein de la communauté des tradipraticiens. Elle est exprimée par le pourcentage de citations d'une espèce par rapport au nombre total de personnes enquêtées. La fréquence de citation de chacun des taxons recensés est calculée par la formule utilisée par Gbekley et al. (2015) et Orsot (2016) :

$$
\mathbf{F C}=\frac{\mathbf{n}}{\mathrm{N}} \times 100
$$

$n:$ nombre de personnes ayant cité l'espèce $N$ : nombre total de personnes interrogées

Traitement des données : Les paramètres recherchés étaient :

- $\quad$ La typologie (types biologiques, morphologiques et phytogéographiques)

- $\quad$ Le Profil des personnes interrogées (âge, sexe et niveau d'instruction)

- $\quad$ Les Parties ou drogues de la plante utilisées

- $\quad$ La Technique de préparation

- $\quad$ La Voie d'administration 


\section{RÉSULTATS}

Étude botanique

Richesse spécifique : Au terme de l'enquête, dix-sept espèces de plantes antidiabétiques ont été recensées dans le Département de Zouénoula. Ces taxons sont repartis en seize genres et quatorze familles botaniques. La famille des Fabaceae avec trois espèces $(17,6 \%)$ est la plus représentée puis, vient la famille des Asteraceae avec deux espèces $(11,7 \%)$. Toutes les autres familles renferment une seule espèce.

\section{Typologie}

Trois types botaniques ont été recherchés dans cette étude.

Types adaptatifs ou biologiques : Les types adaptatifs enregistrés sont au nombre de quatre (Figure 2). Ce sont le thérophytes $(17 \%)$, les chaméphytes $(12 \%)$, les géophytes $(6 \%)$ et phanérophytes $(65 \%)$. Les phanérophytes sont les plus représentés. Les phanérophytes recensés sont constitués des nanophanérophytes, des microphanérophytes et des mésophanérophytes.

Types morphologiques: La figure 3 présente les quatre types morphologiques recensés. Ce sont les arbres ( $18 \%$ ), les lianes $(18 \%)$, les arbustes $(35 \%)$ et les herbes $(29 \%)$. Les arbustes sont les plus représentés.

Types Phytogéographiques : Les espèces répertoriées sont de trois types phytogéographiques. On a celles de la région de forêt dense humide ou Guinéo-Congolaise (GC) avec $35 \%$, les espèces introduite (i) qui représentent $18 \%$ et les taxons de la région Guinéo-Congolaise et SoudanoZambézienne (GC-SZ) avec une représentativité de 47\% (Figure 4). Ces derniers taxons sont majoritairement représentés.

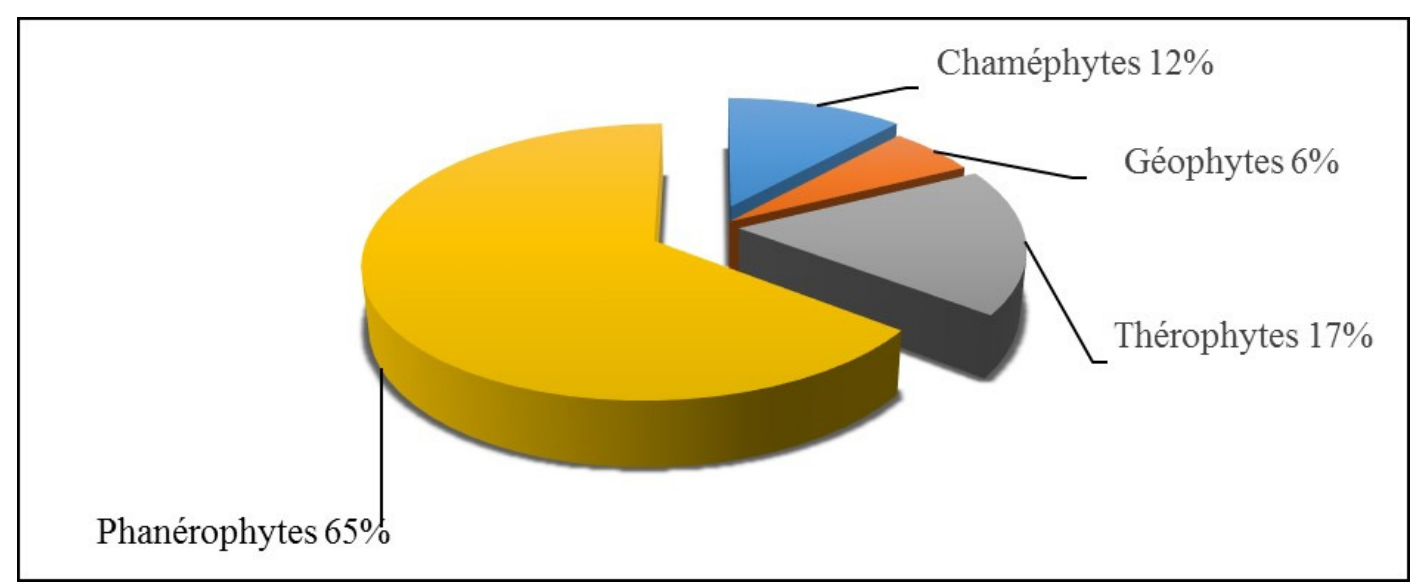

Figure 2 : Spectres des types biologiques

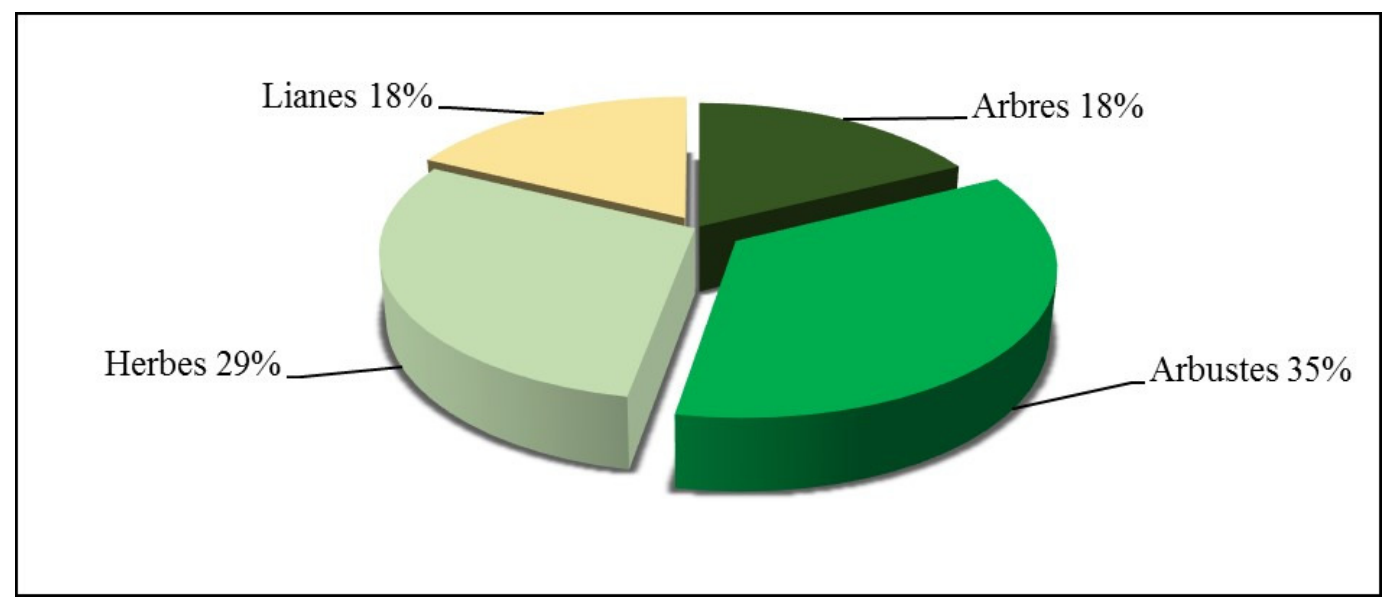

Figure 3 : Spectre des types morphologiques 


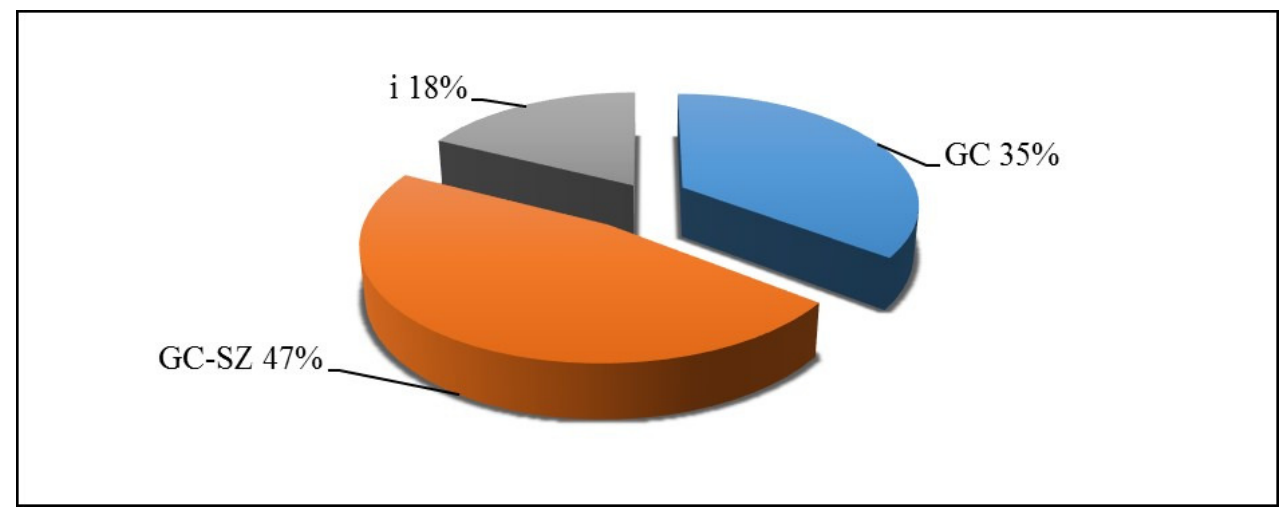

Figure 4 : Spectre des types phytogéographiques

GC : Guinéo-Congolaise,GC-SZ : Guinéo-Congolaise et Soudano-Zambézienne, i : introduite.

Étude ethnobotanique

Répartition selon le sexe : Dans le Département de Zouénoula, cent personnes ayant des connaissances ethnobotaniques ont été enquêtées. Parmi elles, on retrouve aussi bien les hommes que les femmes. Mais, ce sont les hommes au nombre de 65 soit $65 \%$ qui sont les plus représentées (Tableau 1). Les femmes sont moins représentées avec $35 \%$.

Répartition selon l'âge: Les tranches d'âge enregistrées sont : la tranche inférieure à 40 ans, celle comprise entre 40 et 60 ans et enfin la tranche supérieure à 60 ans (Tableau 1). Dans le Département de Zouénoula les tradithérapeutes ont majoritairement un âge supérieur à 60 ans (59\%).

Niveau d'instruction des phytothérapeutes: Concernant le niveau d'instruction des phytothérapeutes, les résultats obtenus montrent que $64 \%$ n'ont pas été scolarisés, alors que $18 \%$ ont un niveau primaire et $15 \%$ ont un niveau secondaire. Les universitaires par contre sont très peu représentés $3 \%$ (Tableau 1).

Parties des plantes utilisées: Trois types d'organes sont utilisés comme drogues : les rameaux feuillés avec $64,7 \%$, les écorces de tige $29,4 \%$ et les bulbes avec $5,9 \%$ (Figure 7). Les résultats indiquent que les rameaux de feuilles sont les parties les plus employées dans la préparation des recettes antidiabétiques dans le Département de Zouénoula.

Techniques de préparation: Pour faciliter l'administration des drogues, trois techniques de préparation sont employées à savoir: la décoction $(88,2 \%)$, la macération $(5,9 \%)$ et la mastication $(5,9 \%)$ (Figure 8).

Voie d'administration : La voie orale est la seule voie d'administration des phytomédicaments antidiabétiques dans le Département de Zouénoula. Le tableau 2 présente la liste générale des espèces antidiabétiques recensées ainsi que les différentes typologies.

Tableau 1 : Informations générales sur les herboristes enquêtés

\begin{tabular}{|c|c|c|c|c|c|}
\hline \multirow{2}{*}{ Paramètres } & \multicolumn{3}{|c|}{ Différentes strates } & \multirow[b]{2}{*}{ Strate 4} & \multirow{2}{*}{ Moyenne } \\
\hline & Strate 1 & Strate 2 & Strate 3 & & \\
\hline Effectif & 25 & 25 & 25 & 25 & 100 \\
\hline \multicolumn{6}{|c|}{ Répartition par sexe (\%) } \\
\hline Homme & 72 & 44 & 60 & 84 & 65 \\
\hline Femme & 28 & 56 & 40 & 16 & 35 \\
\hline \multicolumn{6}{|c|}{ Age (\%) } \\
\hline$<40$ ans & 12 & 4 & 20 & 20 & 14 \\
\hline $40 \leq$ Age $<60$ & 16 & 40 & 32 & 20 & 27 \\
\hline$\geq 60$ ans & 72 & 56 & 48 & 60 & 59 \\
\hline \multicolumn{6}{|c|}{ Niveau d'instruction (\%) } \\
\hline Non scolarisé & 72 & 56 & 48 & 80 & 64 \\
\hline Primaire & 12 & 24 & 32 & 4 & 18 \\
\hline Secondaire & 12 & 20 & 12 & 16 & 15 \\
\hline Supérieur & 4 & 0 & 8 & 0 & 3 \\
\hline
\end{tabular}




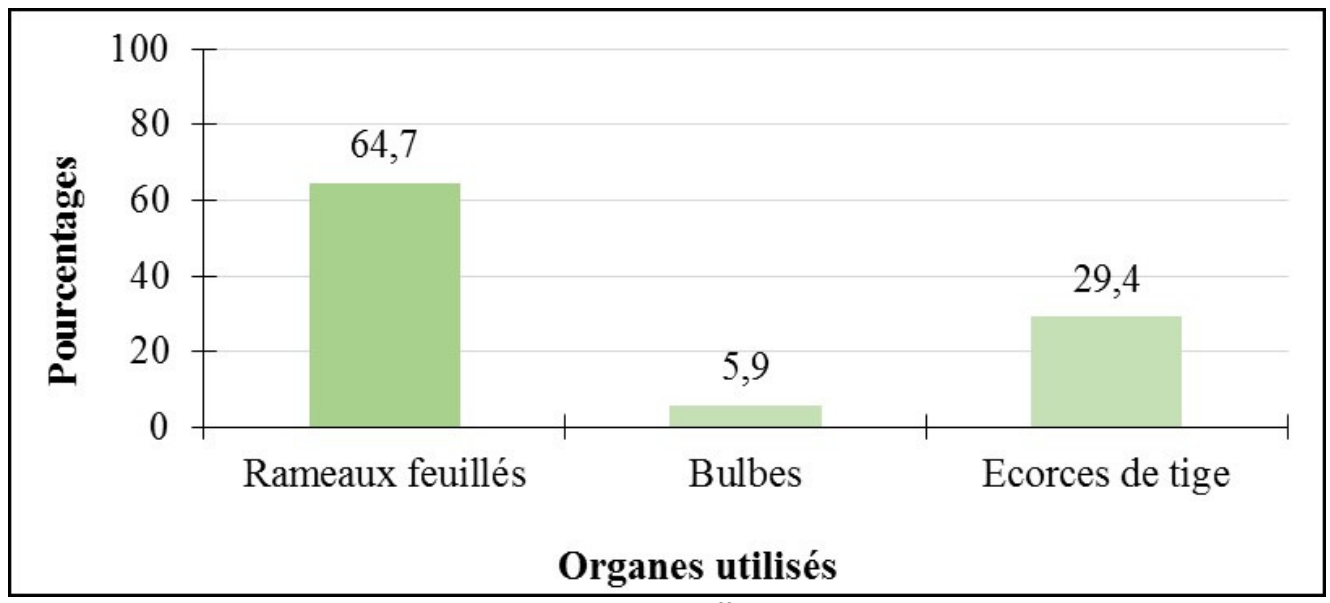

Figure 5 : Histogrammes des différentes parties utilisées

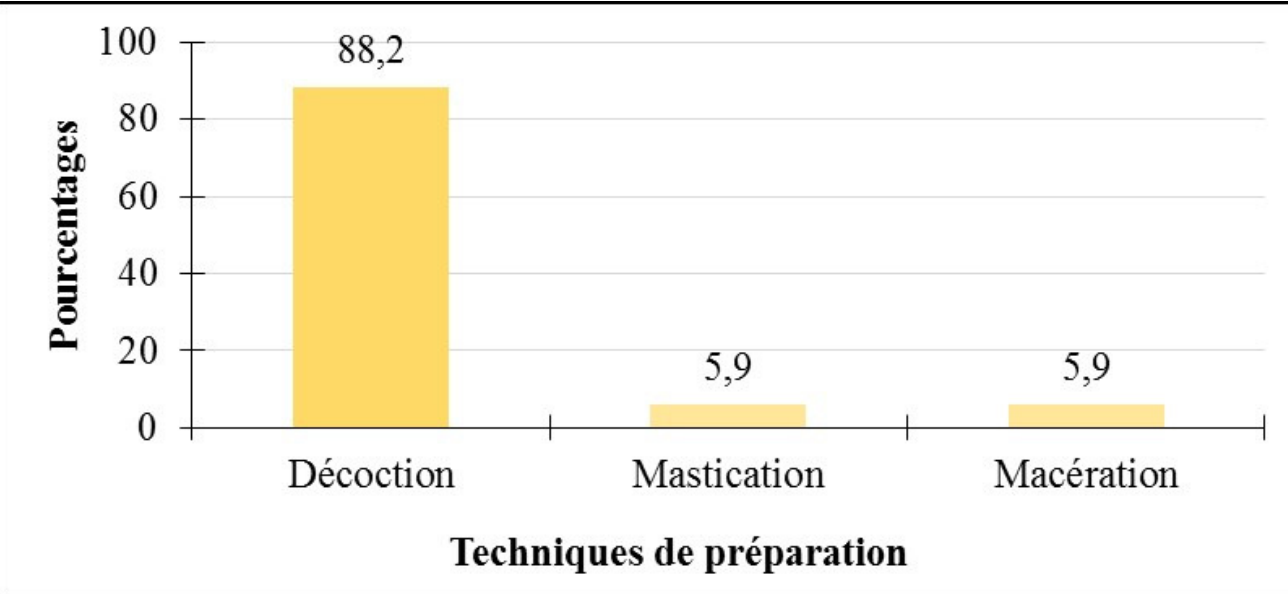

Figure 6 : Histogrammes des techniques de préparation 
Gnagne et al., J. Appl. Biosci. 2017 Étude ethnobotanique des plantes médicinales utilisées dans le traitement du diabète dans le Département de Zouénoula (Côte d'lvoire)

Tableau 2 : Les plantes médicinales antidiabétiques du Département de Zouénoula

\begin{tabular}{|c|c|c|c|c|c|c|c|}
\hline Noms scientifiques & Familles & Biol. & Mph. & Chorologie & Parties utilisées & $\begin{array}{l}\text { Technique de } \\
\text { préparation }\end{array}$ & $\begin{array}{c}\text { Fréquence de } \\
\text { citation (\%) }\end{array}$ \\
\hline Abrus precatorius $\mathrm{L}$. & Fabaceae & $\mathrm{mp}$ & 1 & GC-SZ & Rameaux feuillés & Décoction & 19 \\
\hline Ageratum conyzoides L. & Asteraceae & Th & $\mathrm{h}$ & GC-SZ & Rameaux feuillés & Décoction & 31 \\
\hline Allium sativum $\mathrm{L}$. & Amaryllidaceae & G & $\mathrm{h}$ & $\mathrm{i}$ & Bulbe & Mastication & 15 \\
\hline Anacardium occidentale L. & Anacardiaceae & $\mathrm{mp}$ & $\mathrm{b}$ & $\mathrm{i}$ & Écorces de tige & Décoction & 15 \\
\hline Anthocleista djalonensis A. Chev. & Gentianaceae & $\mathrm{mP}$ & a & GC-SZ & Écorces de tige & Décoction & 37 \\
\hline Bidens pilosa L. & Asteraceae & Th & $\mathrm{h}$ & GC-SZ & Rameaux feuillés & Décoction & 48 \\
\hline Boerhavia diffusa L. & Nyctaginaceae & $\mathrm{Ch}$ & $\mathrm{h}$ & GC-SZ & Rameaux feuillés & Décoction & 13 \\
\hline Cassia occidentalis L. & Fabaceae & $\mathrm{np}$ & $b$ & GC-SZ & Rameaux feuillés & Décoction & 13 \\
\hline Cassia siamea Lam. & Fabaceae & $\mathrm{mp}$ & $\mathrm{b}$ & GC-SZ & Écorces de tige & Décoction & 21 \\
\hline Dissotis rotundifolia (Sm.) Triana & Melastomataceae & $\mathrm{Ch}$ & $\mathrm{h}$ & A GC & Rameaux feuillés & Décoction & 4 \\
\hline Mareya micrantha (Benth.) Müll. Arg. & Euphorbiaceae & $m p$ & $\mathrm{~b}$ & A GC & Rameaux feuillés & Décoction & 7 \\
\hline Momordica charantia L. & Cucurbitaceae & Th & I & GC & Rameaux feuillés & Macération & 16 \\
\hline Morinda morindoides (Bak.) Milne-Redh. & Rubiaceae & $\mathrm{mp}$ & I & GC & Rameaux feuillés & Décoction & 17 \\
\hline $\begin{array}{l}\text { Tapinanthus bengwensis (Engl. \& Krause) } \\
\text { Danser }\end{array}$ & Loranthaceae & $\mathrm{mp}$ & $b$ & GC & Rameaux feuillés & Décoction & 9 \\
\hline Terminalia catappa L. & Combretaceae & $m p$ & $a$ & $\mathrm{i}$ & Écorces de tige & Décoction & 5 \\
\hline Trema guineensis Schum. \& Thonn. & Cannabaceae & $\mathrm{mp}$ & $\mathrm{b}$ & GC-SZ & Rameaux feuillés & Décoction & 11 \\
\hline Zanthoxylum gilletii (Engl.) Waterman & Rutaceae & $\mathrm{mP}$ & a & GC & Écorces de tige & Décoction & 12 \\
\hline
\end{tabular}

Sélection des espèces de plantes : À partir des critères de sélection prédéfinis, trois espèces médicinales ont été retenues. Ce sont : Ageratum conyzoides (31\%), Anthocleista djalonensis (37\%) et Bidens pilosa (48\%). 
Gnagne et al., J. Appl. Biosci. 2017 Étude ethnobotanique des plantes médicinales utilisées dans le traitement du diabète dans le Département de Zouénoula (Côte d'Ivoire)

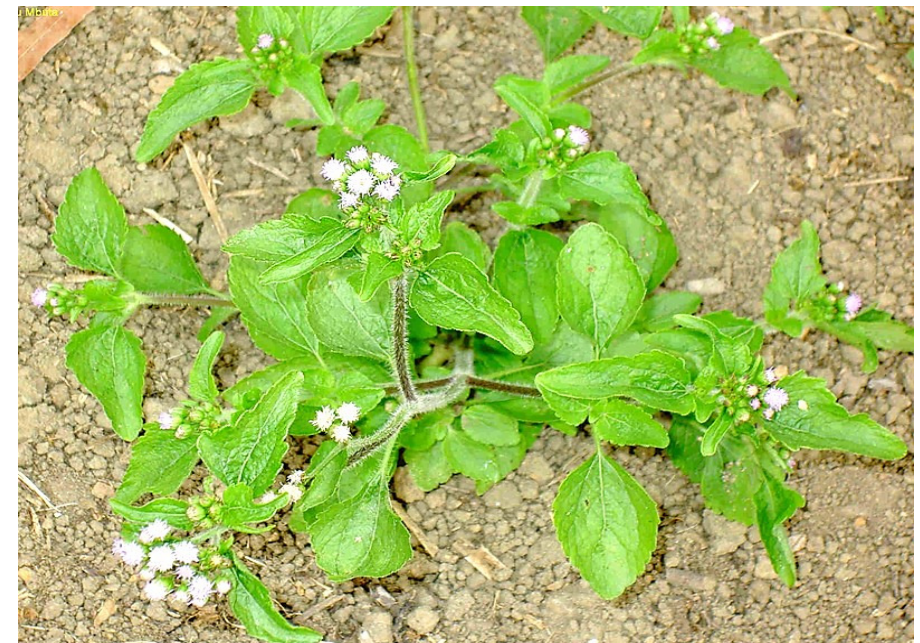

Figure 7 : Ageratum conyzoides L.

(Source : http://www. nzenzeflowerspauwels.be/AgerCony.jpg)

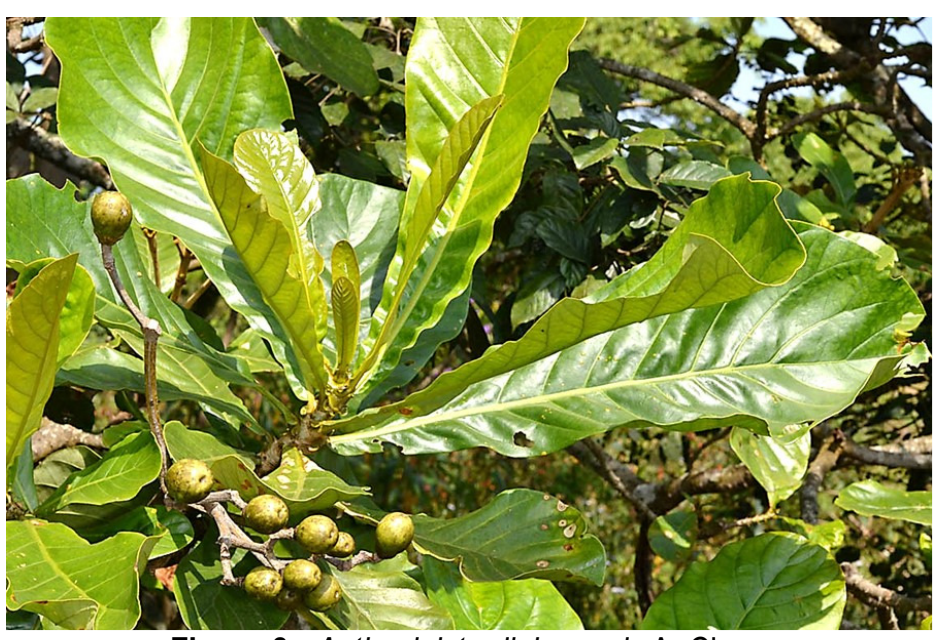

Figure 8 : Anthocleista djalonensis A. Chev.

(Source : http://www.phytoimages.siu.edu/users/pelserpb/2_7_13_3/8feb13/MJW_1141.jpg)

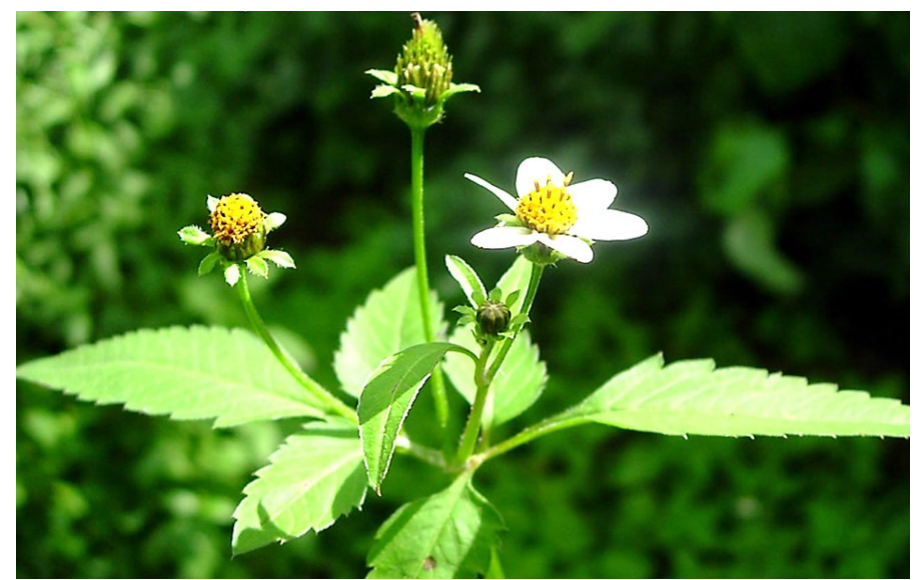

Figure 9 : Bidens pilosa L.

(Source : http://www.westafricanplants.senckenberg.de/images/pictures/aste_bidens_pilosa) 


\section{DISCUSSION}

Les enquêtes ethnobotaniques réalisées sur le terrain ont permis d'interroger 100 tradipraticiens du Département de Zouénoula, qui étaient majoritairement de sexe masculin $(65 \%)$. Ce profil des tradipraticiens est celui observé dans la plupart des études du genre (Gbékley et al., 2015), confirmant que la pratique de la médecine traditionnelle est l'apanage des hommes d'âge mûr. En effet, la majorité des tradithérapeutes (59\%) ont un âge supérieur à 60 ans. Les personnes âgées sont censées fournir des informations plus fiables, du fait qu'elles détiennent une bonne partie du savoir ancestral qui se transmet oralement. La transmission de cette connaissance est en danger actuellement parce qu'elle n'est pas toujours assurée (Orch et al., 2015). II ressort de cette étude que $64 \%$ des personnes enquêtés n'ont pas été scolarisés et ces résultats sont proches des données nationales et montrent que l'usage des plantes médicinales reste l'apanage des personnes pauvres. La forte représentativité des Fabaceae $(17,6 \%)$ pourrait se justifier par le regroupement de trois sous-familles en une famille botanique. En effet, la classification phyllogénétique a associé les Caesalpinioideae, les Mimosoideae et les Faboideae ou Papilionoideae. Elles sont toutes de l'ordre des Fabales (APG III, 2009) et cet ensemble constitue la famille des Fabaceae. Parmi les espèces recensées, les phanérophytes sont les plus nombreux. Ces résultats reflètent l'état de la végétation des zones tropicales et équatoriales, dont la proportion en phanérophytes est estimée entre 80 et $90 \%$ (Ambé et al., 2015). En ce qui concerne les types morphologiques, la forte fréquence des arbustes (35\%) s'expliquerait par le fait que le Département est une zone de transition entre la forêt et la savane. Dans un tel milieu, les arbustes se rencontrent fréquemment dans l'environnement immédiat des utilisateurs et l'on a une facilité d'accès aux organes. Les proportions de la distribution phytogéographique des

\section{CONCLUSION}

L'enquête effectuée dans le Département de Zouénoula a permis de recenser dix-sept espèces médicinales utilisées couramment dans le traitement du diabète. De cette liste de plantes, Ageratum conyzoides, Anthocleista djalonensis et Bidens pilosa sont les plus recommandées.

\section{REMERCIEMENTS}

Nous tenons à remercier le Centre National de Floristique (CNF) de l'Université Félix Houphouët- espèces recensées mettent en évidence la prédominance des espèces de la zone mixte et Soudano-Zambézienne (GC-SZ). En effet, cette dominance pourrait se justifier par le fait que le Département de Zouénoula est situé dans une transition de deux zones phytogéographiques que sont la zone Guinéo-Congolaise (GC) et la zone Soudano-Zambézienne (SZ). L'utilisation fréquente des feuilles est justifiée par l'abondance de groupes chimiques antidiabétiques qu'elles contiennent. Elles sont connues comme le lieu de synthèse des métabolites secondaires chez la plante (Mangambu et al., 2014), mais également par l'aisance et la rapidité de la récolte. Tous ces organes sont préparés principalement sous la forme de décocté. Cela s'explique par le fait que la décoction permet de recueillir le plus de principes actifs et atténue ou annule l'effet toxique de certaines recettes (Salhi et al., 2010). Ces préparations médicamenteuses antidiabétiques sont toutes prescrites par la voie orale et en boisson dans le Département de Zouénoula. Cette prescription peut s'expliquer par le fait que cette pathologie est liée à des organes profonds. Pour les atteindre, tout composé doit transiter par l'appareil digestif pour en faciliter son assimilation (Tra Bi et al., 2008). Ageratum conyzoides, Anthocleista djalonensis et Bidens pilosa sont les trois espèces les plus sollicités dans les préparations antidiabétiques dans le Département de Zouénoula. Les travaux de recherche de Tra Bi et al. (2008) ont confirmé l'usage traditionnel de Ageratum conyzoides et Anthocleista djalonensis dans le traitement du diabète. En outre, ces auteurs ont révélé que les feuilles et la décoction étaient respectivement les parties utilisées et la technique de préparation recommandées dans le traitement de cette pathologie. Lee et al. 2013, ont quant à eux attesté l'usage antidiabétique de Bidens pilosa dans leur étude.

Une étude pharmacologique future permettra d'évaluer l'efficacité thérapeutique et l'innocuité de ces trois plantes à effet antidiabétique traditionnel afin de formuler un Médicament Traditionnel Amélioré (MTA).

Boigny de Cocody-Abidjan pour l'identification et la confirmation des noms des espèces végétales. 


\section{RÉFÉRENCES BIBLIOGRAPHIQUES}

Ambé A.S.A., Ouattara D., Tiebre M.-S., Vroh B.T.A., Zirihi G.N., N'Guessan K.E., 2015. Diversité des plantes médicinales utilisées dans le traitement traditionnel de la diarrhée sur les marchés d'Abidjan (Côte d'Ivoire). Journal of Animal \& Plant Sciences, 26(2) : 4081-4096.

APG III, 2009. The Angiosperm Phylogeny Group, « An update of the Angiosperm Phylog-eny Group classification for the orders and families of flowering plants : APG III », Bo-tanical Journal of the Linnean Society, 161(2) : 105-121.

Chase M.W. \& Reveal J.L., 2009.- «A phylogenetic classification of the land plants to accompany APG III», Botanical Journal of the Linnean Society, $161:$ 122-127.

Fah L., Klotoé J.R., Dougnon V., Koudokpon H., Fanou V.B.A., Dandjesso C. \& Loko F., 2013. Étude ethnobotanique des plantes utilisées dans le traitement du diabète chez les femmes enceintes à Cotonou et Abomey-Calavi (Bénin). Journal of Animal \& Plant Sciences, 18(1) : 2647-2658.

Gbekley E.H., Karou D.S., Gnoula C., Agbodeka K., Anani K., Tchacondo T., Agbonon A., Batawila K., Simpore J., 2015. Étude ethnobotanique des plantes utilisées dans le traitement du diabète dans la médecine traditionnelle de la région Maritime du Togo. PanAfrican Medicla Journal. $20: 437-452$.

Ghourri M., Zidane L., Douira A., 2013. Usage des plantes médicinales dans le traitement du Diabète Au Sahara marocain (Tan -Tan). Journal of Animal \&Plant Sciences, 17(1) : 23882411.

Kroa E., Doh S.K., Soko Y.N., K.S.Yohou, Koulaï O.J.J.D., Gbogbo M., N'Guessan K., Aka J. \& Kouassi D., 2016. Effet de l'extrait aqueux de l'écorce de tige de Anthocleista djalonensis A. Chev. (Gentianaceae) sur la glycémie des lapins. Int. J. Biol. Chem. Sci. 10(2): 552-558.

Lee W.-C., Peng C.-C., Chang C.-H., Huang S.-H. \& Chyau C.-C., 2013. Extraction of Antioxidant Components from Bidens pilosa Flowers and Their Uptake by Human Intestinal Caco-2 Cells. Molecules, 18: 1582-1601.

Mangambu M.J.de.D., Mushagalusa K.F. \& Kadima N.J., 2014. Contribution à l'étude phytochimique de quelques plantes médicinales antidiabétiques de la ville de Bukavu et ses environs (Sud-Kivu,
R.D. Congo). Journal of Applied Biosciences, 75 :6211-6220.

O.M.S, 2011 : Diabète. Aide-mémoire №312, septembre 2011.

Orch H., Douira A. \& Zidane L., 2015. Étude ethnobotanique des plantes médicinales utilisées dans le traitement du diabète, et des maladies cardiaques dans la région d'Izarène (Nord du Maroc). Journal of Applied Biosciences, 86 : 7940-7956.

Orsot B.A.M.B., 2016.- Étude ethnobotanique des plantes médicinales utilisées dans le traitement des maladies de la peau par les Abbey du Département d'Agboville (Côte d'Ivoire) et évaluation de l'activité antifongique des extraits de quatre plantes sur Scle-rotium rolfsii, un phytopathogène. Thèse de Doctorat Unique de Botanique, Université Félix HOUPHOUËTBOIGNY, Côte d'Ivoire, 168p.

Salhi S., Fadli M., Zidane L. \& Douira A., 2010. Études floristique et ethnobotanique des plantes médicinales de la ville de Kénitra (Maroc). Lazaroa 31 : 133-146.

SANOFI, 2014. Le Diabète une épidémie mondiale. 16p. Tahri N., El Basti A., Zidane L., Rochdi A. \& Douira A., 2012. Étude Ethnobotanique des plantes médicinales dans la Province se Settat (Maroc). J. Forest. Fac., 12(2) : 192-208.

Tra Bi F.H., Irié G.M., N'gaman K.C.C. \& Mohou C.H.B., 2008. Études de quelques plantes thérapeutiques utilisées dans le traitement de l'hypertension artérielle et du diabète : deux maladies émergentes en Côte d'Ivoire. Sciences \& Nature 5(1) : 39-48. 